\title{
ACPT gene is inactivated in mammalian lineages that lack enamel or teeth
}

\author{
Yuan Mu ${ }^{1}$, Xin Huang ${ }^{1}$, Rui Liu ${ }^{1}$, Yulin Gai ${ }^{1}$, Na Liang ${ }^{1}$, Daiqing Yin ${ }^{1}$, Lei Shan ${ }^{1}$, Shixia Xu ${ }^{1}$, Guang Yang ${ }^{\text {Corresp. } 1}$ \\ 1 Jiangsu Key Laboratory for Biodiversity and Biotechnology, College of Life Sciences, Nanjing Normal University, Nanjing, Jiangsu, China \\ Corresponding Author: Guang Yang \\ Email address: gyang@njnu.edu.cn
}

Loss of tooth or enamel is widespread in multiple mammal lineages. Although several studies have been reported, the evolutionary mechanisms of tooth / enamel loss are still unclear. Most previous studies have found that some tooth-related genes have been inactivated in toothless and / or enamel-less mammals, such as ENAM, ODAM, C4orf26, $A M B N, A M T N, D S P P$, etc. Here, we conducted evolutionary analyses on ACPT playing a key role in amelogenesis, to interrogate the mechanisms. We obtained the ACPT sequences from 116 species, including edentulous and enamel-less mammals. The results shows that variant ORF-disrupting mutations were detected in ACPT coding region among nine edentulous baleen whales and three enamel-less taxa (pygmy sperm whale, aardvark, nine-banded armadillo). Furtherly, selective pressure uncovered that the selective constraints have been relaxed among all toothless and enamel-less lineages. Moreover, our results support the hypothesis that mineralized teeth were lost or degenerated in the common ancestor of crown Mysticeti through two shared single-base sites deletion in exon 4 and 5 of $A C P T$ among all living baleen whales. $D_{N} / d_{S}$ values on transitional branches were used to estimate ACPT inactivation records. In the case of aardvark, inactivation of ACPT was estimated at $\sim 23.60-28.32 \mathrm{Ma}$, which is earlier than oldest aardvark fossil record (Orycteropus minutus, $\sim 19 \mathrm{Ma}$ ), suggesting that $A C P T$ inactivation may result in degeneration or loss of enamel. Conversely, the inactivation time of ACPT estimated in armadillo $(\sim 10.18-11.30 \mathrm{Ma})$ is later than oldest fossil record, suggesting that inactivation of ACPT may result from degeneration or loss of enamel in these mammals. Our findings suggested that different mechanisms of degeneration of tooth / enamel might exist among toothless and enamel-less lineages during evolution. Our study further considered that $A C P T$ is a novel gene for studying tooth evolution. 


\section{ACPT gene is inactivated in mammalian lineages that lack enamel or} 2 teeth

3

4 Yuan Mu${ }^{1}$, Xin Huang ${ }^{1}$, Rui Liu ${ }^{1}$, Yulin Gai ${ }^{1}$, Na Liang ${ }^{1}$, Daiqing Yin ${ }^{1}$, Lei Shan ${ }^{1}$, Shixia Xu ${ }^{1}$

5 and Guang Yang ${ }^{1 *}$

6

$7 \quad{ }^{1}$ Jiangsu Key Laboratory for Biodiversity and Biotechnology, College of Life Sciences, Nanjing

8 Normal University, Nanjing 210023, China

9

$10 *$ Corresponding author:

11 Guang Yang

12 1\# Wenyuan Road, Nanjing, Jiangsu Province, 210023, China

13 Email address: gyang@njnu.edu.cn

14 


\section{Abstract}

Loss of tooth or enamel is widespread in multiple mammal lineages. Although several studies have been reported, the evolutionary mechanisms of tooth / enamel loss are still unclear. Most previous studies have found that some tooth-related genes have been inactivated in toothless and / or enamel-less mammals, such as ENAM, ODAM, C4orf26, AMBN, AMTN, DSPP, etc. Here, we conducted evolutionary analyses on $A C P T$ playing a key role in amelogenesis, to interrogate the mechanisms. We obtained the $A C P T$ sequences from 116 species, including edentulous and enamel-less mammals. The results shows that variant ORF-disrupting mutations were detected in $A C P T$ coding region among nine edentulous baleen whales and three enamel-less taxa (pygmy sperm whale, aardvark, nine-banded armadillo). Furtherly, selective pressure uncovered that the selective constraints have been relaxed among all toothless and enamel-less lineages. Moreover, our results support the hypothesis that mineralized teeth were lost or degenerated in the common ancestor of crown Mysticeti through two shared single-base sites deletion in exon 4 and 5 of $A C P T$ among all living baleen whales. $D_{\mathrm{N}} / d_{\mathrm{S}}$ values on transitional branches were used to estimate $A C P T$ inactivation records. In the case of aardvark, inactivation of $A C P T$ was estimated at $\sim 23.60-28.32 \mathrm{Ma}$, which is earlier than oldest aardvark fossil record (Orycteropus minutus, $\sim 19 \mathrm{Ma}$ ), suggesting that $A C P T$ inactivation may result in degeneration or loss of enamel. Conversely, the inactivation time of $A C P T$ estimated in armadillo ( 10.18-11.30 Ma) is later than oldest fossil record, suggesting that inactivation of $A C P T$ may result from degeneration or loss of enamel in these mammals. Our findings suggested that different mechanisms of degeneration of tooth / enamel might exist among toothless and enamel-less lineages during evolution. Our study further considered that $A C P T$ is a novel gene for studying tooth evolution. Key works: $A C P T$, Tooth evolution, Enamel loss, Mammals, Pseudogene, Inactivation time 
39

40

41

\section{Introduction}

Dental innovations (such as differentiated dentitions and the evolution of tri-bosphenic molar) have been regarded as the great success of mammalian evolution and adaptation (Ungar, 2010). However, in spite of their importance for animal survival, teeth have been lost independently in multiple mammalian lineages, such as baleen whales and pangolins. In addition, some lineages have lost their outer enamel of teeth, such as pygmy sperm whale and dwarf sperm whale, aardvarks and species from Xenarthra (Davitbeal et al., 2009). Tooth loss and / or enamel loss is one of the most important field for mammalian tooth evolution.

Amelogenesis imperfecta (AI) and tooth loss are the diseases that characterized by genetic defects in the formation of enamel and teeth. Multiple studies have suggested these genetic disorders are mainly caused by mutations of protein-coding genes functioned in formation of enamel and teeth (Stephanopoulos et al., 2005; Smith et al., 2017). Of these genes, three enamel matrix protein genes (EMPs, i.e., $A M E L X, A M B N$ and $E N A M$ ), two enamel proteases genes (MMP20 and KLK4), and some other related genes (e.g., C4orf26, AMTN, ODAM, ACPT, DSPP) have been confirmed to be candidate genes responsible for the diseases (Crawford et al., 2007; Smith et al., 2017). The variant inactivating mutations have been detected in these genes among toothless and enamel-less mammalian lineages. However, the mechanisms of tooth loss or enamel loss are still completely unclear.

It has been reported that $A C P T$ was lower expressed in testicular cancer tissues compared to normal tissues and is regulated by steroid hormones (Yousef et al., 2001). Besides, $A C P T$ is also expressed in the brain and acts as a tyrosine phosphatase to modulate signals mediated by ErbB4 (Fleisig et al., 2004). But, it is interesting to note that $A C P T$ is expressed in secretory-stage ameloblasts (Seymen et al., 2016), which can induce odontoblasts differentiation, mineralization of dentin, and amelogenesis (Choi et al., 2016). Furthermore, there are some increasing evidences that homozygous missense variants of $A C P T$ would lead to $\mathrm{AI}$ (e.g., c.226C $>\mathrm{T}$, p.Arg76Cys; c.746C4T, p.P249L) (Seymen et al., 2016; Smith et al., 2017). These evidences suggested that $A C P T$ play an important role in amelogenesis.

All extant Mysticeti, descended from toothed ancestors, have no teeth and instead have baleen (Uhen, 2010). Paleontological evidences have shown that mineralized teeth were lost in the common ancestor of crown Mysticeti. Moreover, a transitional stage from tooth to baleen in stem mysticetes have been revealed in some taxa bearing both teeth and baleen (Deméré et al., 
2008). Although many tooth-related genes have been revealed to be inactivated in various living mysticetes (e.g., AMBN, ENAM, AMEL, AMTN, MMP20, C4orf26 and DSPP) (Deméré et al., 2008; Meredith et al., 2009; Meredith et al., 2011; Gasse et al., 2012; Delsuc et al., 2015; Springer et al., 2016; Springer et al., 2019), only the MMP20 are commonly inactivated across all the living baleen whales (Meredith et al., 2011). This molecular evidence is consistent with earlier studies of paleontology and anatomy.

Despite its significance in mammalian enamel maturation, very little is known about $A C P T$ evolutionary trajectory, relationship and function in mammals. To address this issue, we carried out a series of evolutionary analyses on $A C P T$, aim to uncover the evolutionary pattern of $A C P T$ gene among mammalian lineages.

\section{Methods}

\section{Sequences mining and BLAST searches}

The full-length coding sequences (CDS) of $A C P T$ gene were extracted from the OrthoMaM v10b (http://orthomam2.mbb.univ-montp2.fr/OrthoMaM_v10b10/), ENSEMBL (http://www.ensembl.org/index.html?redirect=no) and NCBI (http://www.ensembl.org/index.html?redirect=no) databases (Table S1). ACPT of some whales were extracted from their Genome and SRA database of NCBI (Table S2 and S3). To further ensure the sites of inactivating mutation of toothless / enamel-less lineages, we used the CDSs of some representative placental species with well-annotated genomes (Homo sapiens [human], Canis lupus familiaris [Dog], Bos taurus [Cow], Echinops telfairi [Lesser hedgehog tenrec]) as queries including $\sim 50 \mathrm{bp}$ of flanking sequence on each exon. These sequences were used as queries to BLAST against toothless / enamel-less mammals to confirm the related inactivating mutation among baleen whales.

\section{Identification of inactivating mutations and functional sites and domains}

The intact $A C P T$ sequences (human, cow, tenrec) were used for identifying inactivating mutations (including mutation of initiation codons, frame-shift insertions and deletions, premature stop codons, splice sites mutation of intron / exon boundary [GT/AG], etc.). The inactivating mutation was identified based on BLAST searches against whole genomes of the relevant taxon from NCBI. The information on gene function, related key amino acid sites/domains was searched from UniProtKB/Swiss-Prot (http://www.uniprot.org/) and some references. 
101

102

103

104

105

106

107

108

109

110

111

112

113

114

115

116

117

118

119

120

121

122

123

124

125

126

127

\section{Alignment and phylogenetic analysis of mammalian $A C P T$}

The 116 mammalian $A C P T$ sequences were aligned based on their amino acid translations using online PRANK (https://www.ebi.ac.uk/goldman-srv/webprank/), and then deleted the gaps and non-homologous regions by using GBLOCK, then we corrected the multiple sequences alignment (MSA) in MEGA 7 (Kumar et al., 2016) by eye.

A gene tree was reconstructed by Mrbayes 3.2 (Ronquist et al., 2012) with a general time reversible (GTR) substitution model and rate heterogeneity modeled with a Gamma distribution, as conducted by MrModeltest version 2 using the Akaike information criterion (AIC) (Nylander, 2004). In bayesian analysis, four simultaneous runs with four chains each were run for two million generations, sampling every 1000 trees. The first $25 \%$ of these trees were discarded as burn-in when computing the consensus tree. Tracer v1.5 software was used for checking convergence among chains in Bayesian analysis. When the ESS value is higher than 200, and the average standard deviation of spilt frequencies is lower than 0.01 , we think it reach convergence level.

\section{Selection analyses}

To evaluate the selective pressure of relevant branches leading to enamel-less and toothless lineages respectively, we implemented two ratio branch model to calculate the ratio of the nonsynonymous substitution rate $\left(d_{\mathrm{N}}\right)$ to the synonymous substitution rate $\left(d_{\mathrm{S}}\right)\left(\omega=d_{\mathrm{N}} / d_{\mathrm{S}}\right)$ by running CodeML in PAML 4.8a package (Yang, 2007). We also recoded premature stop codons as missing data. Akaike information criterion (AIC) scores were used to select the most appropriate codon frequency model in CodeML. The $A C P T$ gene tree exhibits different topological relationship compared to species tree, which may be unrelated to incomplete lineage sorting. In order to illuminate the detected signal reasonably and accurately, we used a species tree supported by some previous studies (Fig. S1).

Refer to the methods of Springer and Gatesy (Springer and Gatesy, 2018), several different branch categories were considered during selective analyses: (1) One category accounted for 'background' branches, which are lineages with intact teeth and an intact copy of $A C P T$. (2) Nine branch categories to terminal branches with unique inactivating mutations (baleen whales), which lacks teeth. (3) Three branch categories to terminal branches with unique inactivating mutations (pygmy sperm whale, nine-banded armadillo and aardvark), whose enamel has been vestigial. (4) One branch categories were assigned for stem Mysticeti where mineralized teeth 
132

133

134

135

136

137

138

139

140

141

142

143

144

145

146

147

148

149

150

151

152

153

154

155

156

157

158

159

160

161

were degraded. (5) One branch categories were assigned for crown Mysticeti.

To better understand the selective pressure, a series of evolutionary models were compared in the likelihood. We first use the M0 model (Model A), which assumed that all branches in the phylogenetic tree has a common value, and compare it with the null hypothesis (Model B), which assumed that the common value in the phylogenetic tree is 1 . To further understand whether the selective pressure on the lineages leading to pseudogenes was relaxed, we constructed Model C, which assumed that the branches with pseudogene had their own selection pressure $\omega_{2}$, while the background branches without pseudogenization was $\omega_{1}$, and then compared Model C with Model A. To further confirm whether the selective pressure on the lineages leading to pseudogenes was completely relaxed, we build the Model D, which assumed that the branches with pseudogene had their own selection pressure $\omega_{2}=1$, while the selective pressure of background branches was $\omega_{1}$, and then compared Model C with Model D.

\section{Estimation of inactivation times}

To estimate when $A C P T$ was inactivated in different lineages of Placentalia, the method described in Chou et al. (2003) and Zhang et al. (2010) was used. Among the branches along which the gene became pseudogenes, this method presumes that gene evolves under a selective pressure similar to that in other species until it is inactivated. Next, this gene was presumed to accumulate both nonsynonymous and synonymous mutations at an equal rate. The $\mathrm{K}_{\mathrm{a}} / \mathrm{K}_{\mathrm{s}}(\mathrm{K})$ value was assessed for this entire branch. The average $\mathrm{K}_{\mathrm{a}} / \mathrm{K}_{\mathrm{s}}$ value was just for a part of the branch, where the gene was under selection (Ks). In addition, the $\mathrm{K}_{\mathrm{a}} / \mathrm{K}_{\mathrm{s}}$ value for the rest of part of the branch where the gene evolved neutrally $\left(K_{n}=1\right)$. Thus, the evolutionary time was weighted by the proportion, for which the gene was evolving under selection $\left(\mathrm{T}_{\mathrm{s}} / \mathrm{T}\right)$ and neutrally $\left(\mathrm{T}_{\mathrm{n}} / \mathrm{T}\right)$ :

$\mathrm{K}=\mathrm{K}_{\mathrm{s}} \times \mathrm{T}_{\mathrm{s}} / \mathrm{T}+\mathrm{K}_{\mathrm{n}} \times \mathrm{T}_{\mathrm{n}} / \mathrm{T}$

where $\mathrm{T}$ is the time since the split from the last common ancestor (LCA). By selecting the lower and upper bound of the confidence interval for the species divergence time $\mathrm{T}$, which was obtained from TimeTree website (http://www.timetree.org/) to estimate a lower and upper bound for $T_{n}$ as:

$\mathrm{T}_{\mathrm{n}}=\mathrm{T} \times\left(\mathrm{K}-\mathrm{K}_{\mathrm{s}}\right) /\left(1-\mathrm{K}_{\mathrm{s}}\right)$

which provides an estimate of how long the $A C P T$ gene has been evolving neutrally. 


\section{Results}

\section{Characterization of $\boldsymbol{A C P T}$ sequence}

164120 sequences were obtained in this study. Due to the poor quality and low coverage of 165 sequences among three pangolins (Manis javanica, M. javanica, Phataginus tricuspis) and one 166 sloth (Choloepus hoffmanni), they were not used for subsequent analysis. However, some

\section{7} inactivating mutations (most of them are indels) were found in these sequences (Fig. S2). The complete protein-coding sequence of $A C P T$ in 116 taxa were used for alignment by PRANK. Interestingly, one or more inactivating mutations (frame-shift mutation, initial codon mutation, premature stop codons, splice site mutations, etc.) were detected in another placental taxa without teeth or without enamel. (Fig. 1, Table S4, Fig. S3). For example, among toothless baleen whales, the initial codon mutation ( $\mathrm{n} . \mathrm{ATG} \rightarrow \mathrm{GTG}, \mathrm{p} . \mathrm{M} \rightarrow \mathrm{V}$ ) was found in Balaenoptera borealis, B. physalus, B. musculus, Eschrichtius robustus, Eubalaena glacialis. Meanwhile, premature stop codons were found in B. acutorostrata and B. bonaerensis, frameshift indels were also found in baleen whales. Interestingly, two shared single-base site deletion was found on exon 4 and 5 of $A C P T$ among all living baleen whales (Fig. 1, Fig. S3). The splice site mutations were detected in B. acutorostrata, Eubalaena japonica and Megaptera novaeangliae (Table S4). Whilst, the premature stop codons were found in enamel-less D. novemcinctus and . afer. Besides, frameshift indels were found in enamel-less Kogia breviceps.

Except for the species mentioned above, $A C P T$ gene in other species whose teeth are intact were found to be activated. Nevertheless, some crucial amino acids mutation was found in toothed species, such as site 76 has been mutated (R76C) in Neophocaena asiaeorientalis.

\section{Reconstruction of $A C P T$ gene tree}

We recovered the $A C P T$ gene tree with well-supported values by using Mrbayes method (Fig. 2). In this gene tree, most of orders have been well reconstructed, and have high support rate, e.g., Cetartiodactyla, Perissodactyla, Eulipotyphla, Carnivora, Chiroptera etc. In addition, phylogenetic relationships of higher levels have also been well reconstructed, such as Laurasiatheria, Euarchontoglires, Boreoeutheria and Afrotheria. In this gene tree, bayesian posterior probability (PP) values of nearly $70 \%$ nodes are generally greater than 0.70 . However, the relationship between some order level were relatively chaotic, such as Lagomorpha didn't cluster with Rodentia, but as the sister group of Primate; Chiroptera and Carnivora clustered together first, and then they became sister group of Perissodactyla. 
193

194

195

196

197

198

199

200

201

202

203

204

205

206

207

208

209

210

211

212

213

214

215

216

217

218

219

220

221

222

223

\section{Evolutionary analyses among toothless and enamel-less mammals}

We carried out the PAML analysis to detect the selective pressure of toothless / enamel-less lineages, and found the selective pressure of these toothless / enamel-less lineages (including ancestral nodes, terminal branches and even the whole toothless / enamel-less group) was significantly higher than that of background branches. For example, the terminal branch of $B$. physalus: $\omega_{1}=0.116, \omega_{2}=1.883$; the terminal branch of $M$. novaeangliae: $\omega_{1}=0.116, \omega_{2}=0.641$; the terminal branch of E. robustus: $\omega_{1}=0.116, \omega_{2}=2.688$; the terminal branch of E. glacialis: $\omega_{1}=0.116, \omega_{2}=0.503$. A similar tendency was found in the terminal branches of other baleen whales, and further model comparison shows that the selective pressure of these branches had been completely relaxed. Whilst, much higher selective pressure was detected in the ancestral branch of stem mysticeti $\left(\omega_{1}=0.120, \omega_{2}=0.436\right)$, even the clade of crown mysticeti $\left(\omega_{1}=0.116\right.$, $\left.\omega_{2}=0.522\right)$. Meanwhile, higher selective pressure was detected among enamel-less lineages, such as the terminal branch of $D$. novemcinctus $\left(\omega_{1}=0.116, \omega_{2}=0.206\right)$, the terminal branch of $O$. afer $\left(\omega_{1}=0.116, \omega_{2}=0.414\right)$, and the terminal branch of $K$. breviceps $\left(\omega_{1}=0.116, \omega_{2}=0.581\right)$. And the selective pressure of these branches had been completely relaxed, except for the terminal branch of $K$. breviceps (Table S5).

\section{$A C P T$ inactivation dates}

Estimates of inactivation times for $A C P T$ based on $d_{\mathrm{N}} / d_{\mathrm{S}}$ ratios and equations in Sharma et al. (Sharma et al., 2018). The mean estimate for the inactivating time of $A C P T$ on the branch of $K$. breviceps, D. novemcinctus and $O$. afer is $12.20-15.52 \mathrm{Ma}, 10.18-11.30 \mathrm{Ma}$ and $23.60-28.32 \mathrm{Ma}$, respectively (Fig. 3). The mean estimate for the inactivation of $A C P T$ on the Mysticeti clade is 14.05-16.30Ma.

\section{Discussion}

\section{$A C P T$ is a novel candidate gene for studying mammalian tooth loss and enamel loss}

The well-conserved gene structure in extant species indicates that this organization and arrangement might be present in the last common mammalian ancestor, which represented the vital function for organisms (Madsen, 2009). In our study, the number of $A C P T$ exons are 11 in placental mammals, which encode 427 amino acids (human ACPT sequence as the reference sequence). Our study collected that four residues (191N, 269N, 330N and 339N) of the extracellular region were for glycosylation, two residues $(41 \mathrm{H}$ and $289 \mathrm{D})$ directly involved in catalysis (from UniProt database). In addition, mutation in seven residues were reported that 
224 were responsible for AI (Seymen et al., 2016; Smith et al., 2017) (Fig. S4). Besides, there are

225 three disulfide bond regions, namely, site 159 to 378 , site 214 to 312, site 353 to 357 . In fact, we

226

227

228

229

230

231

232

233

234

235

236

237

238

239

240

241

242

243

244

245

246

247

248

249

250

251

252

253

254

detected not only teratogenic mutations but also inactivated mutations in these functional sites and domains. For example, enamel in finless porpoise were degenerated (Ishiyama, 1987), mutation in site $76(\mathrm{R} \rightarrow \mathrm{C})$ was found in $N$. asiaeorientalis. Previous research has confirmed that site 76 mutated into Cys (C) in human ACPT would lead to hypoplastic AI (Seymen et al., 2016), from which this result further supported that teeth in finless porpoise were degenerated in molecular level. Of cause, most obvious characteristics of $A C P T$ is that different types of inactivating mutations were found in toothless and enamel-less mammals, e.g., baleen whales, pangolins, sloths and so on (Fig. S2, Fig. S3). Therefore, ACPT could be a candidate gene for AI and studying mammalian tooth loss and enamel loss.

\section{Degeneration or loss of mineralized teeth in LCA of Mysticeti}

Fossil evidence shows that the earliest ancestors of baleen whales possessed complete dentitions without baleen (such as Janjucetus and Mammalodon), and then evolved the baleen with teeth (such as Aetiocetus), until the lineages only baleen existed (e.g., Eomysticetus and Micromysticetus) (Fitzgerald, 2006; Fitzgerald, 2010; Meredith et al., 2011). However, the fact is all living baleen whales lack teeth and instead baleen (Uhen, 2010). This implied that that mineralized teeth were lost or degenerated gradually in the common ancestors of all modern baleen whales (Boessenecker and Fordyce, 2015). In addition, the successive steps of vestigial tooth development was found in the fetal period of living baleen whales (Davit-Béal et al., 2009; Thewissen, 2018), which was also confirmed by genetic evidence. Molecular sequences of some specific genes, such as $A M B N, E N A M, A M E L X, A M T N, C 4 o r f 26$ and $O D A M$, contain different types of inactivating mutations (e.g., stop codons, frameshift mutations, splice site mutations, etc.) in various mysticete species (Deméré et al., 2008; Meredith et al., 2009; Alhashimi et al., 2010; Gasse et al., 2012; Meredith et al., 2013; Delsuc et al., 2015; Springer et al., 2019), which is consistent with loss-of-teeth in this group. But none of the inactivating mutations are shared by all living mysticetes species. Meredith et al. found a common insertion of CHR-2 SINE retroposon in MMP20 gene among all living baleen whales (Meredith et al., 2011). Previous study has been confirmed that mutations or deletions of MMP20 gene would result in thin and brittle enamel layer (Caterina et al., 2002). Based on this result, they confirmed the hypothesis that mineralized teeth were lost or degenerated in the common ancestor of crown Mysticeti in the

Peer) reviewing PDF | (2020:03:46572:2:1:NEW 28 Sep 2020) 
255

256

257

258

259

260

261

262

263

264

265

266

267

268

269

270

271

272

273

274

275

276

277

278

279

280

281

282

283

284

285

molecular level.

In this research, we also identified different inactivating mutations was detected among all mysticete species in $A C P T$ gene, among which two shared single-base sites deletion were found on exon 4 and 5 of $A C P T$ among all living baleen whales, which result in loss of function. Some studies have confirmed that $A C P T$ gene is responsible for the development of enamel, and mutations can also lead to amelogenesis imperfecta (Choi et al., 2016; Seymen et al., 2016; Smith et al., 2017). Similar to the result of Meredith et al. (2011), our study supported the hypothesis that mineralized teeth were lost or degenerated in the common ancestor of all extant baleen whales.

\section{Is inactivation of $A C P T$ neutral or adaptive?}

The degeneration and / or loss of some morphological structures (such as limbs, teeth, and eyes, etc.) is a complex process that may result from the relaxation of the negative selection (neutral evolution), adaptive evolution (direct natural / positive selection to conserve energy and / or eliminate the disadvantageous effects of morphological structure), and / or gene pleiotropy (indirect selection on another traits) (Wang et al., 2006; Zhang, 2008; Krishnan and Rohner, 2017). In some conditions, evolutionary change also results from differences in the reproductive success of individuals with different genotypes (Olson,1999). Sharma et al. (2018) revealed that evolutionary gene losses are not only a consequence, but may also be causally involved in phenotypic adaptations. By estimating the inactivation time of pseudogenes, and comparing with oldest fossil records, we might be able to speculate whether gene inactivation is due to the adaptive or neutral selection after the loss of phenotype.

The record of enamel-degenerated armadillo fossil is significantly earlier than the estimated time of $A C P T$ inactivation (10.18-11.30Ma) (Ciancio et al., 2014), which suggested gene loss as a consequence of adaptation is likely the result of the relaxation of the negative selection. The results further supported the previous study (Sharma et al., 2018). Besides, during the tooth evolution, some enamel-related genes (e.g., ODAM, ENAM, $A M B N$ ) also have gone through the similar evolutionary trajectory. By integrating different results from different methods, we may better understand the evolution of teeth and enamel. The inactivation time of ENAM ( 45.5Ma) and $O D A M(\sim 40.43 \mathrm{Ma}$, range $36.38-45.45 \mathrm{Ma})$ is much earlier than inactivation date for $A C P T$ in armadillo (Springer et al., 2019). $A C P T$ inactivation is later than the fossil record, conversely, the inactivation time of $E N A M$ is relatively earlier than the fossil record, which implied the 
286

287

288

289

290

291

292

293

294

295

296

297

298

299

300

301

302

303

304

305

306

307

308

309

310

311

312

313

314

315

316

various mechanisms of enamel loss in armadillo. Here, the inactivation of ENAM gene might be the causes of degeneration / loss of tooth enamel in armadillos, $A C P T$ inactivation might be the consequence of enamel loss.

For $O$. afer, even the inactivation date for $A C P T(23.60-28.32 \mathrm{Ma})$ is relatively younger than inactivation dates for ENAM (28.8-35.3Ma) and $O D A M(\sim 30.7 \mathrm{Ma})$ in $O$. afer (Meredith et al., 2009; Springer et al., 2019). However, the estimated inactivation times by $A C P T, O D A M$ and ENAM gene markers are all earlier than the oldest fossil record of aardvark (O. minutus, $19 \mathrm{Ma})$ (Patterson, 1975). It should be suggested that gene loss may be the reason, not the consequence, for degeneration and / or loss of enamel. Moreover, due to the difference of species number, sequences quality and topological structure of species tree, the result of $A C P T$ inactivation time is different from the result of Sharma et al. (Sharma et al., 2018).

Cetacean includes both toothless Mysticeti and enamel-less Kogia. Relaxation of selective pressure was detected in both crown and stem Mysticeti (Table S5), which is consistent with the archaic toothless mysticete, namely, all stem Mysticeti were toothless. For example, Eomysticetus whitmorei, an edentulous species, was the geologically oldest mysticete (Deméré et al., 2008). Molecular evidence shows $A C P T$ has been lost its function in LCA of Mysticeti. However, the inactivation time of $A C P T$ in Mysticeti is $14.05-16.30 \mathrm{Ma}$, which is much younger than the toothless mysticete ( 30Ma) and the split of Mysticeti ( 25.9Ma). Obviously, this is not consistent with the facts. It might be associated with relatively lower rates of frameshift accumulation during evolution of mysticete pseudogenes and long lifespan of mysticete (Meredith et al., 2009; Meredith et al., 2011). Whether adaptive or neutral, the shared single-base site deletion in $A C P T$ fills an important gap in our understanding of the macroevolutionary transition leading from the LCA of crown Cetacean to the LCA of crown Mysticeti. Stem physeteroids (sperm whales) are known from the Miocene and had teeth with enamel (Bianucci and Landini, 2010). Our results provide support for loss of the intact ACPT in K. breviceps. ACPT was reported that play key roles in amelogenesis and differentiation of odontoblasts (Choi et al., 2016; Seymen et al., 2016; Smith et al., 2017). Our result is in line with the enamel-less morphological structure in K. breviceps.

\section{CONCLUSIONS}

We detected the different types of inactivated mutation in $A C P T$. Furthermore, selective pressure uncovered that the selective constraints have been relaxed among all toothless and enamel-less 
317 lineages. In addition, our results supported the hypothesis that mineralized teeth were lost or

318 degenerated in the common ancestor of crown Mysticeti through two shared single-base sites

319 deletion in exon 4 and 5 of $A C P T$ among all living baleen whales. Together with our evidence,

320 ACPT might be a good marker to research the mechanism of tooth loss. By comparing the

321 molecular time with the fossil time, we found there might be different mechanisms of

322 degeneration of tooth / among toothless and enamel-less lineages during evolution, which is

323 needed further researches.

\section{Acknowledgements}

325 We thank members of the Jiangsu Key Laboratory for Biodiversity and Biotechnology, Nanjing

326 Normal University, for their contributions to this paper. The authors thank Mr. Xinrong Xu, Dr.

327 Di Sun and Dr. Ran Tian, Dr. Zepeng Zhang, Dr. Simin Chai and Dr. Zhenpeng Yu for some

328 helpful discussion. Special thanks to Dr. Zhengfei Wang for technical supports. 


\section{References}

330

331

Alhashimi N, Lafont AG, Delgado S, Kawasaki K, Sire JY. 2010. The enamelin genes in lizard, crocodile, and frog and the pseudogene in the chicken provide new insights on enamelin evolution in tetrapods. Molecular Biology and Evolution 27:2078-2094.

Bianucci G, Landini W. 2010. Killer sperm whale: a new basal physeteroid (Mammalia, Cetacea) from the Late Miocene of Italy. Zoological Journal of the Linnean Society 148:103-131.

Boessenecker RW, Fordyce RE. 2015. Anatomy, feeding ecology, and ontogeny of a transitional baleen whale: a new genus and species of Eomysticetidae (Mammalia: Cetacea) from the Oligocene of New Zealand. PeerJ 3:e1129.

Caterina JJ, Skobe Z, Shi J, Ding Y, Simmer JP, Birkedalhansen H, Bartlett JD. 2002. Enamelysin (Matrix Metalloproteinase 20) deficient mice display an amelogenesis imperfecta phenotype. Journal of Biological Chemistry 277:49598-49604.

Choi H, Kim TH, Yun CY, Kim JW, Cho ES. 2016. Testicular acid phosphatase induces odontoblast differentiation and mineralization. Cell and Tissue Research 364:95-103.

Chou HH, Hayakawa T, Diaz S, Krings M, Indriati E, Leakey M, Paabo S, Satta Y, Takahata N, Varki A. 2002. Inactivation of CMP- $N$-acetylneuraminic acid hydroxylase occurred prior to brain expansion during human evolution. Proceedings of the National Academy of Sciences of the United States of America 99:11736-11741.

Ciancio MR, Vieytes EC, Carlini AA. 2014. When xenarthrans had enamel: insights on the evolution of their hypsodonty and paleontological support for independent evolution in armadillos. Naturwissenschaften 101:715-725.

Crawford PJ, Aldred M, Bloch-Zupan A. 2007. Amelogenesis imperfecta. Orphanet Journal of Rare Diseases 2:17.

Davit-Béal T, Tucker AS, Sire JY. 2009. Loss of teeth and enamel in tetrapods: fossil record, genetic data and morphological adaptations. Journal of Anatomy 214:477-501.

Delsuc F, Gasse B, Sire JY. 2015. Evolutionary analysis of selective constraints identifies ameloblastin $(\mathrm{AMBN})$ as a potential candidate for amelogenesis imperfecta. BMC Evolutionary Biology 15:148.

Deméré TA, Mcgowen MR, Berta A, Gatesy J. 2008. Morphological and molecular evidence for a stepwise evolutionary transition from teeth to baleen in mysticete whales. Systematic 
360

361

362

363

364

365

366

367

368

369

370

371

372

373

374

375

376

377

378

379

380

381

382

383

384

385

386

387

388

389

390

Biology 57:15-37.

Fitzgerald EM. 2006. A bizarre new toothed mysticete (Cetacea) from Australia and the early evolution of baleen whales. Proceedings of the Royal Society B: Biological Sciences 273:2955-2963.

Fitzgerald EMG. 2010. The morphology and systematics of Mammalodon colliveri (Cetacea: Mysticeti), a toothed mysticete from the Oligocene of Australia. Zoological Journal of the Linnean Society 158:367-476.

Fleisig H, El-Husseini ED, Vincent SR. 2004. Regulation of ErbB4 phosphorylation and cleavage by a novel histidine acid phosphatase. Neuroscience 127:91-100.

Gasse B, Silvent J, Sire JY. 2012. Evolutionary analysis suggests that AMTN is enamelspecific and a candidate for AI. Journal of Dental Research 91:1085.

Ishiyama M. 1987. Enamel structure in odontocete whales. Scanning microscopy 1:1071-1079.

Krishnan J, Rohner N. 2017. Cavefish and the basis for eye loss. Philosophical Transactions of The Royal Society B Biological Sciences 372:20150487.

Kumar S, Stecher G, Tamura K. 2016. MEGA7: Molecular Evolutionary Genetics Analysis version 7.0 for bigger datasets. Molecular Biology and Evolution 33:1870-1874.

Madsen O. 2009. Mammals. In: The time tree of life. New York, NY: Oxford University Press.

Meredith RW, Gatesy J, Cheng J, Springer MS. 2011. Pseudogenization of the tooth gene enamelysin (MMP20) in the common ancestor of extant baleen whales. Proceedings of the Royal Society B: Biological Sciences 278:993-1002.

Meredith RW, Gatesy J, Murphy WJ, Ryder OA, Springer MS. 2009. Molecular decay of the tooth gene enamelin (ENAM) mirrors the loss of enamel in the fossil record of placental mammals. PLoS Genetics 5:e1000634.

Meredith RW, Gatesy J, Springer MS. 2013. Molecular decay of enamel matrix protein genes in turtles and other edentulous amniotes. BMC Evolutionary Biology 13:20.

Nylander JAA. 2004. MrModeltest v2. Program distributed by the author. Evolutionary Biology Centre, Uppsala University.

Olson MV. 1999. When Less Is More: Gene Loss as an Engine of Evolutionary Change[J]. American Journal of Human Genetics 64:18-23.

Patterson B. 1975. The fossil aardvarks (Mammalia: Tubulidentata). Bulletin of the Museum of Comparative Zoology 147:185-237. 
391

392

393

394

395

396

397

398

399

400

401

402

403

404

405

406

407

408

409

410

411

412

413

414

415

416

417

418

419

420

421

Ronquist F, Teslenko M, Der Mark PV, Ayres DL, Darling AE, Hohna S, Larget B, Liu L, Suchard MA, Huelsenbeck JP. 2012. MrBayes 3.2: Efficient Bayesian Phylogenetic Inference and Model Choice across a Large Model Space. Systematic Biology 61:539-542.

Seymen F, Kim YJ, Lee YJ, Kang J, Kim TH, Choi H, Koruyucu M, Kasimoglu Y, Tuna EB, Gencay K. 2016. Recessive mutations in $A C P T$, encoding testicular acid phosphatase, cause hypoplastic amelogenesis imperfecta. The American Journal of Human Genetics 99:1199-1205.

Sharma V, Hecker N, Roscito JG, Foerster L, Langer BE, Hiller M. 2018. A genomics approach reveals insights into the importance of gene losses for mammalian adaptations. Nature Communications 9:1215.

Smith CE, Whitehouse LL, Poulter JA, Brookes SJ, Day PF, Soldani F, Kirkham J, Inglehearn CF, Mighell AJ. 2017. Defects in the acid phosphatase ACPT cause recessive hypoplastic amelogenesis imperfecta. European Journal of Human Genetics 25:1015-1019.

Smith CEL, Poulter JA, Antanaviciute A, Kirkham J, Brookes SJ, Inglehearn CF, Mighell AJ. 2017. Amelogenesis imperfecta: genes, proteins, and pathways. Frontiers in Physiology 8:435.

Springer M, Emerling C, Gatesy J, Randall J, Collin M, Hecker N, Hiller M, Delsuc F. 2019. Odontogenic ameloblast-associated $(O D A M)$ is inactivated in toothless/enamelless placental mammals and toothed whales. BMC Evolutionary Biology 19:31.

Springer MS, Gatesy J. 2018. Evolution of the $M C 5 R$ gene in placental mammals with evidence for its inactivation in multiple lineages that lack sebaceous glands. Molecular Phylogenetics and Evolution 120:364-374.

Springer MS, Starrett J, Morin PA, Lanzetti A, Hayashi C, Gatesy J. 2016. Inactivation of C4orf26 in toothless placental mammals. Molecular Phylogenetics and Evolution 95:34-45.

Stephanopoulos G, Garefalaki ME, Lyroudia K. 2005. Genes and related proteins involved in amelogenesis imperfecta. Journal of Dental Research 84:1117.

Thewissen JGM. 2018. Highlights of cetacean embryology. Aquatic Mammals 44:591-602.

Uhen MD. 2010. The Origin(s) of Whales. Annual Review of Earth and Planetary Sciences 38:189-219.

Ungar PS. 2010. Mammal teeth: origin, evolution, and diversity: Johns Hopkins University Press, Baltimore, MD. 
422 Wang X, Grus WE, Zhang J. 2006. Gene losses during human origins. PLoS Biology 4:e52.

423 Yang Z. 2007. PAML 4: Phylogenetic Analysis by Maximum Likelihood. Molecular Biology $424 \quad$ and Evolution 24:1586-1591.

425 Yousef GM, Diamandis M, Jung K, Diamandis EP. 2001. Molecular cloning of a novel 426 human acid phosphatase gene $(A C P T)$ that is highly expressed in the testis. Genomics $427 \quad 74: 385-395$.

428 Zhang JZ. 2008. Positive selection, not negative selection, in the pseudogenization of resA in 429 Yersinia pestis. Proceedings of the National Academy of Sciences 105:E69. unitary pseudogenes: historic and contemporary gene losses in humans and other primates. Genome Biology 11:R26.

433

434 
Figure 1

The inactivating mutation of $A C P T$ gene in toothless/enamel-less mammals.

ICM, initiation codon mutation; Del, deletion; Ins, insertion; PSC, premature stop codon. The images are from the PHYLOPIC database: http://phylopic.org/.

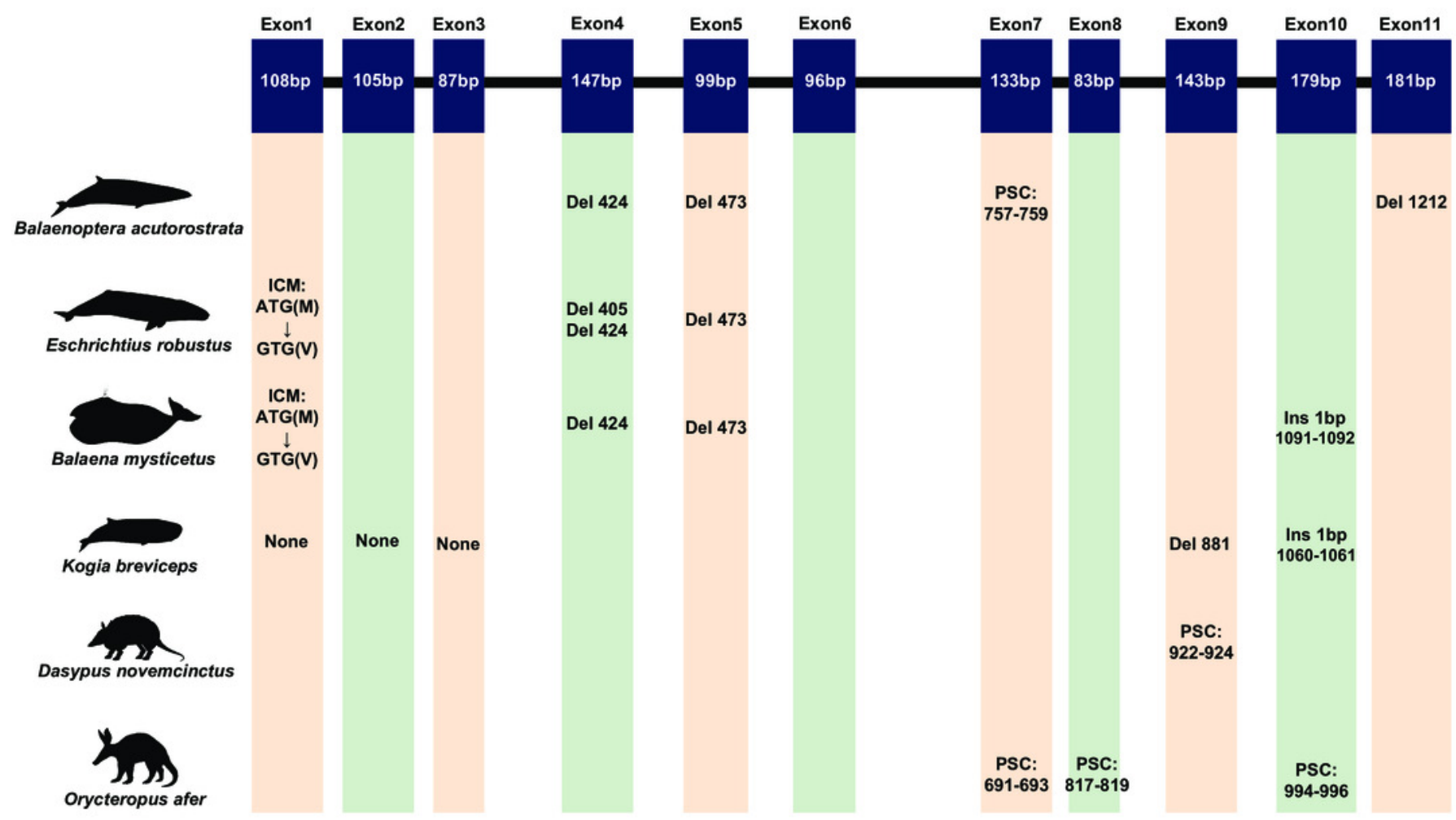


Figure 2

The BI phylogenetic relationship of mammalian ACPT gene used in this study.

Nucleotide optimal substitution model: GTR+GAMMA; green box indicates toothless taxa, red boxes indicate enamel-less taxa. The images are from the PHYLOPIC database:

http://phylopic.org/.

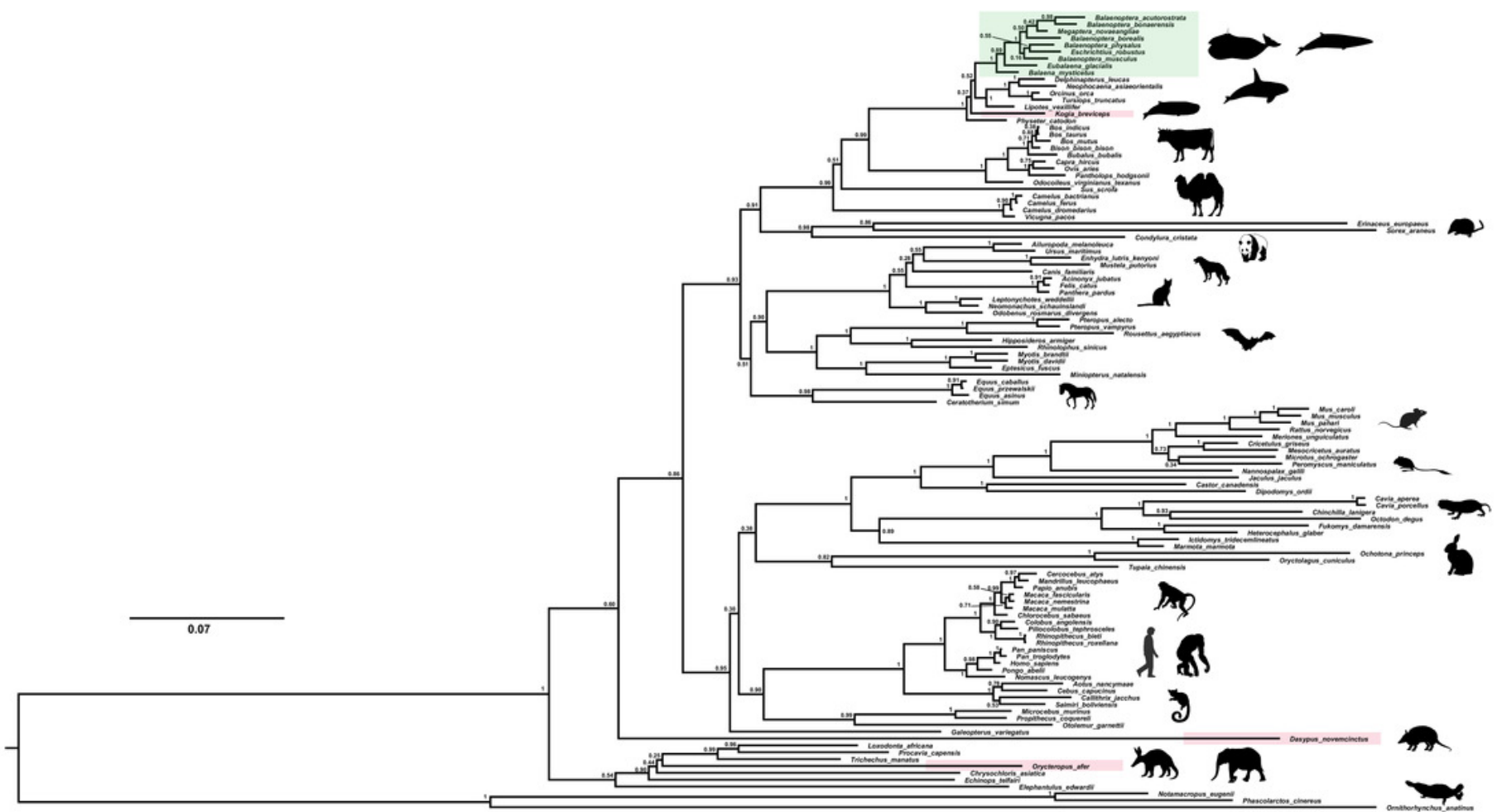


Figure 3

Estimated inactivation times of ACPT versus ENAM.

(a) Dasypus novemcinctus (nine-banded armadillo), (b) Orycteropus afer (aardvark), (c) Kogia breviceps (pygmy spermwhale). The inactivation times of ENAM is from (Meredith et al., 2009; Springer et al., 2019). The images are from the PHYLOPIC database:

http://phylopic.org/. 
a

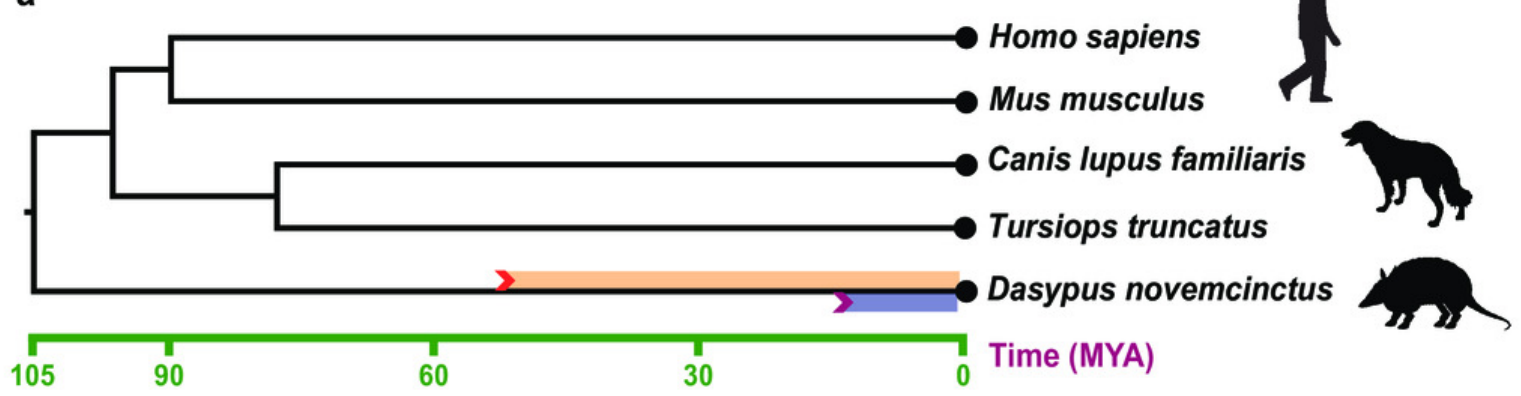

b

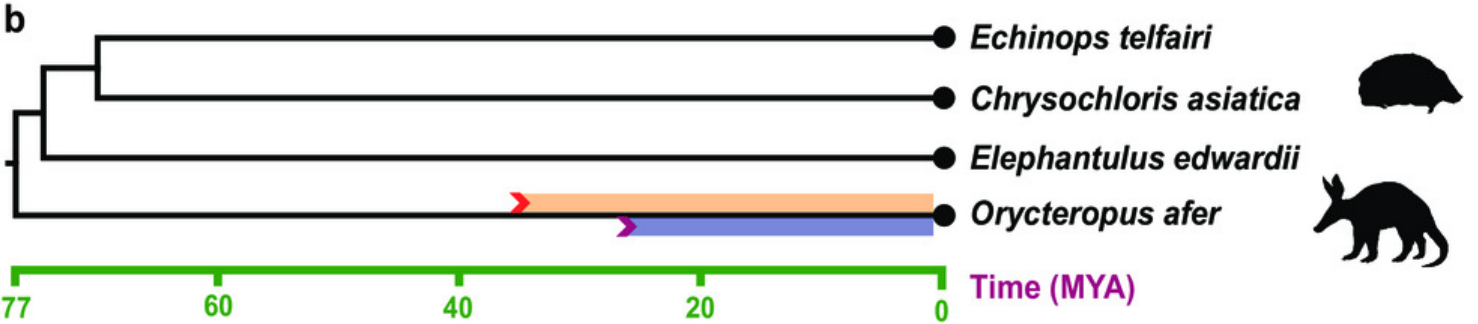

C

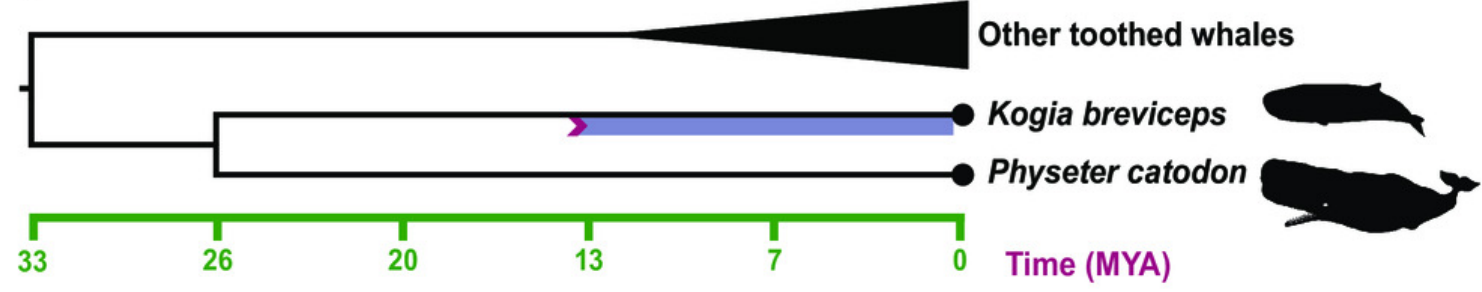

$>=$ initial ENAM inactivation $\quad>=$ initial $A C P T$ inactivation 